\title{
1 eHealth - Wie die digitale Vernetzung unser Gesundheitssystem zukunftssicher macht
}

\author{
Katja Leikert
}

Eine immer größere Zahl von Menschen benutzt mobile Apps zur Analyse von Gesundheitsdaten. Es gibt Pilotprojekte mit Computersystemen, die Klinikärzte bei der Anordnung der richtigen Untersuchungen und Therapien unterstützen; Krankenhäuser stellen, teilweise komplett, auf papierfreies Arbeiten um. Gleichzeitig stellt der Gebrauch von Fax und Brief zur Übermittlung von Behandlungsdokumenten immer noch die Realität in vielen deutschen Arztpraxen und Krankenhäusern dar. Innerhalb dieses Spannungsfeldes vollzieht sich der digitale Wandel im Gesundheitsbereich und bringt viele Veränderungen mit sich. Dabei ist die Digitalisierung beides - Herausforderung und Chance zugleich. Sie bietet uns neue Möglichkeiten zur Gestaltung der Versorgung und zur Verbesserung der Kommunikation. Gleichermaßen sind alle Akteure im Gesundheitswesen gefordert, lang gewachsene Prozesse und Gewohnheiten umzustellen.

\section{Das eHealth-Gesetz - Neuer Impuls für die digitale Vernetzung des Gesundheitswesens}

Die digitale Vernetzung im Gesundheitswesen war lange Zeit durch starre Strukturen und Stillstand gekennzeichnet. Mehr als 70 Millionen gesetzlich Versicherte sind zwar inzwischen im Besitz einer elektronischen Gesundheitskarte, der Nutzen der Karte ist allerdings stark eingeschränkt. Mit dem Gesetz für sichere digitale Kommunikation und Anwendungen im Gesundheitswesen (,eHealth-Gesetz“), das am 3. Dezember 2015 vom Bundestag verabschiedet wurde, ist es gelungen, der Digitalisierung im Gesundheitswesen neue Impulse zu verleihen. Ziel ist es, mithilfe der 
digitalen Vernetzung die Versorgung der Patienten zu verbessern sowie gleichermaßen die Teilhabe und Akzeptanz aller Akteure im Gesundheitswesen sicherzustellen.

Dazu legt das Gesetz einen konkreten Zeitplan für den bundesweiten Aufbau einer sicheren digitalen Infrastruktur des Gesundheitswesens sowie für die Einführung zahlreicher nutzbringender Anwendungen vor. Durch die Verknüpfung mit Sanktionen bestehen nun verbindliche Anreize für eine fristgerechte Umsetzung der Maßnahmen. Erste Meilensteine sind hierbei schon erreicht und haben Eingang in die Versorgung gefunden.

\begin{abstract}
Mit dem eHealth-Gesetz hat der Gesetzgeber seinen Willen zur schnellen digitalen Vernetzung im Gesundheitswesen zum Ausdruck und die entsprechenden Maßnahmen auf den Weg gebracht. Die an Fristen und Sanktionen geknüpfte Umsetzung obliegt nun den Partnern in der Selbstverwaltung.
\end{abstract}

Grundlage dieses nationalen eHealth-Projekts bildet dabei die digitale Vernetzung der 150.00o Arztpraxen, 2.00o Krankenhäuser, 20.00o Apotheken, 2.3 Millionen sonstigen Gesundheitsberufe und der gesetzlich Versicherten durch die Telematikinfrastruktur (TI). Auf Basis dieser Infrastruktur des deutschen Gesundheitswesens wird der Austausch von Daten in Zukunft nicht nur einfacher, sondern auch sicherer. Denn die derzeit überwiegende Praxis der Übermittlung von Daten per Fax oder Brief ist zum einen aufwändiger und aus datenschutzrechtlicher Perspektive zudem ungenügend.

Die erste Anwendung der TI steht bereits in den Startlöchern. Dabei handelt es sich um die automatische Aktualisierung der Versichertenstammdaten (u.a. die Adresse des Versicherten, Krankenkassenzugehörigkeit). Nach erfolgreichen Tests in der Testregion Nordwest wird der bundesweite Rollout der TI voraussichtlich im Herbst dieses Jahres beginnen. Um eine Teilnahme aller Ärzte am Online-Abgleich der Versichertenstammdaten zu gewährleisten, kommt es bei einem Ausbleiben der Teilnahme ab dem 1. Juli 2018 zu pauschalen Kürzungen der Vergütung.

Damit im Ernstfall möglicherweise lebenswichtige Informationen über bestehende Allergien oder Vorerkrankungen sofort verfügbar sind, ist ab 2018 ebenfalls die Speicherung der Notfalldaten auf der elektronischen Gesundheitskarte vorgesehen.

Mit Blick auf die Sicherstellung der Arzneimitteltherapiersicherheit (AMTS) spielt der Medikationsplan eine bedeutende Rolle. Lebensgefährliche Arzneimittelwechselwirkungen können mit seiner Hilfe verhindert werden. Noch immer sterben in Deutschland mehr Menschen aufgrund solcher Wechselwirkungen als im Straßenverkehr. Deshalb haben gesetzlich Versicherte, die gleichzeitig dauerhaft mehr als drei verordnete Arzneimittel anwenden, Anspruch auf die Erstellung eines Medikationsplans durch ihren Arzt. Dabei werden Apotheker, zu deren Kernaufgaben die Sicherstellung der AMTS gehört, von Anfang an einbezogen. Sie sind auf Wunsch des Versicherten zur Aktualisierung des Plans verpflichtet. Dadurch ist eine lückenlose Kontrolle gewährleistet. Darüber hinaus haben die Versicherten ab 2019 Anspruch auf die Aktualisierung ihres elektronischen Medikationsplans bei ihrem Arzt oder Apotheker.

Auch der Einstieg in die Telemedizin ist dank des eHealth-Gesetzes gelungen. Konkret sind im Gesetz zwei Anwendungen verankert. So wurden am 1. April 2017 sowohl 
die telekonsiliarische Befundbeurteilung von Röntgenaufnahmen als auch die Videosprechstunde eingeführt.

Die elektronische Patientenakte (ePA) bildet das Herzstück des eHealth-Gesetzes. Mit ihrer Hilfe können strukturierte Patientendaten digital zusammengeführt und verfügbar gemacht werden. Patienten können damit in Zukunft auf ihre Gesundheitsdaten zugreifen und diese beispielsweise nach einem Umzug oder Arztwechsel ihrem Behandler unkompliziert zur Verfügung stellen. Damit werden nicht nur unnötige Doppelbefundungen vermieden, sondern es wird sichergestellt, dass die für die Versorgung eines Patienten wesentlichen Daten, wenn nötig, am richtigen Ort sind. Das bedeutet vor allem, dass die Daten in die Souveränität des Patienten zurückgehen. Denn die Daten gehören weder dem Krankenhaus, noch dem Arzt. Vielmehr gehören sie dem Patienten und er entscheidet, wer Zugriff darauf hat.

Mit der elektronischen Patientenakte ist es möglich, die strukturierten Patientendaten digital zusammenzuführen und verfügbar zu machen. Die Daten gehören dem Patienten. Er entscheidet darüber, wer Zugriff darauf hat.

\section{Nach dem eHealth-Gesetz ist vor dem eHealth-Gesetz - Zukünftiger Handlungsbedarf und Chancen der digitalen Vernetzung}

Nach dem jahrelangen Stillstand ist das klare politische Bekenntnis zur digitalen Vernetzung unseres Gesundheitswesens ein wichtiges Signal. Inzwischen wird das Thema eHealth von allen Seiten positiv aufgenommen und flankiert. Die Schnelligkeit, mit der beispielsweise einige Krankenkassen inzwischen an digitalen Angeboten für ihre Versicherten arbeiten, hat einen bemerkenswerten Wettbewerb im Markt ausgelöst, von dem letztlich die Versicherten profitieren.

Mit dem eHealth-Gesetz ist das Fundament für die digitale Infrastruktur gelegt. Die darin getroffenen Maßnahmen gilt es fristgerecht umzusetzen und das Projekt zügig zu realisieren. Darüber hinaus sind im Rahmen einer nationalen eHealth-Strategie für Deutschland unter anderem folgende Punkte voranzutreiben.

\section{Stärkung des selbstbestimmten Patienten}

Der digitale Wandel sollte auch weiterhin konsequent dafür genutzt werden, den selbstbestimmten Patienten zu stärken, insbesondere auch im Hinblick auf das ArztPatienten-Verhältnis. An erster Stelle sollte das informationelle Selbstbestimmungsrecht des Patienten stehen. Dafür muss gewährleistet sein, dass der Patient jederzeit über das Internet auf seine in der ePA gespeicherten Gesundheitsdaten zugreifen kann. Dazu gehört auch die Förderung der digitalen Gesundheitskompetenz der Bürger durch die Vermittlung seriöser und wissenschaftlich gesicherter Erkenntnisse. Angesichts zahlreicher im Internet befindlicher Gesundheitsinformationen, die nicht wissenschaftlich belegt sind, bedarf es mehr Aufklärung und Transparenz. Dazu wollen wir ein nationales Gesundheitsportal schaffen, in dem Patienten verlässliche und qualitativ gesicherte Informationen über Krankheiten, Behandlungen sowie die Strukturen unseres Gesundheitssystems finden. 


\section{Die Vernetzung aller Akteure vorantreiben}

Die Vernetzung aller Akteure im Gesundheitswesen muss langfristig auf den Weg gebracht werden. Denn um die Versorgungsstrukturen zukunftsfest zu machen, bedarf es neuer digitaler Lösungen, die sektorenübergreifend funktionieren. So sollte u.a. die Anbindung des Pflegebereichs sowie der Heil- und Hilfsmittelerbringer an die Telematikinfrastruktur schnell umgesetzt werden.

\section{Ausbau der Telemedizin}

Für eine moderne Versorgung sowohl in der Stadt als auch im ländlichen Raum ist die Telemedizin in die Fläche zu bringen. Denn mit ihrer Hilfe kann medizinische Versorgung zu den Menschen gebracht werden, für die diese anderweitig schwer zugänglich ist, weil sie auf dem Land leben oder relativ immobil sind.

\section{Big Data effektiv für die Forschung nutzen}

Die immer größeren Datenmengen, die im Rahmen der Digitalisierung des Gesundheitswesens anfallen, bergen großes Potenzial für die Gesundheitsversorgung und -forschung. Durch die Erhebung und Zusammenführung von Daten, die beispielsweise im Rahmen von Routineuntersuchungen anfallen, können in Zukunft Krankheiten und mögliche Risiken früher und präziser erkannt werden. Derzeit bleiben viele dieser Daten allerdings noch ungenutzt. Zudem ist ein ungehinderter Austausch von Daten zwischen verschiedenen Forschungsstandorten oftmals nicht möglich. Hier gilt es bei gleichzeitiger Wahrung des Datenschutzes, weiter daran zu arbeiten, mehr Daten für die Forschung zugänglich zu machen, um so die bestmögliche Versorgung der Menschen sicherzustellen.

\section{Datenschutz und Datensicherheit}

Gerade im Zeitalter von Big Data ist ein Höchstmaß an Datenschutz und -sicherheit unabdingbar für den Erfolg und die Akzeptanz der digitalen Vernetzung im Gesundheitswesen. Hierbei wird es für alle Beteiligten auch weiterhin wichtig sein, diese nicht als Digitalisierungshemmnisse zu verstehen.

Im Zeitalter von Big Data ist ein Höchstmaß an Datenschutz und -sicherheit Voraussetzung für den Erfolg und die Akzeptanz der digitalen Vernetzung im Gesundheitswesen. Die Sicherheit und der Schutz der Patientendaten müssen zum Gütesiegel der Digitalisierung des deutschen Gesundheitswesens werden.

\section{Fazit und Ausblick}

Mit der Digitalisierung bieten sich vielfältige Chancen für unser Gesundheitswesen. Im Rahmen einer nationalen eHealth-Strategie sollten unsere langfristigen Ziele sowie zentrale Meilensteine formuliert werden. Das heißt vor allem ein höherer Grad 
an Selbstbestimmung durch den Patienten, die langfristige Vernetzung aller Akteure im Gesundheitsbereich sowie die Erschließung neuer Möglichkeiten für die Cesundheitsforschung. Dazu zählt idealerweise auch die administrative Entlastung von Ärzten und Pflegepersonal, sodass mehr Zeit für den Patienten gewonnen wird. Die Digitalisierung stellt keinen Selbstzweck dar, sondern soll zum Nutzen der Patienten und der Leistungserbringer gesteuert werden. Dabei sollte immer das Kernziel unserer Anstrengungen im Zentrum stehen: die kontinuierliche Verbesserung der Versorgung der Patienten. 